\title{
Neuroimaging Findings and Repeat Neuroimaging Value in Pediatric Chronic Ataxia
}

\author{
Michael S Salman, Bernard N Chodirker, Martin Bunge
}

\begin{abstract}
Background: Chronic ataxia, greater than two months in duration, is encountered relatively commonly in clinical pediatric neurology practise and presents with diagnostic challenges. It is caused by multiple and diverse disorders. Our aims were to describe the neuroimaging features and the value of repeat neuroimaging in pediatric chronic ataxia to ascertain their contribution to the diagnosis and management. Materials and Methods: A retrospective charts and neuroimaging reports review was undertaken in 177 children with chronic ataxia. Neuroimaging in 130 of 177 patients was also reviewed. Results: Nineteen patients had head computed tomography only, 103 brain magnetic resonance imaging only, and 55 had both. Abnormalities in the cerebellum or other brain regions were associated with ataxia. Neuroimaging was helpful in 73 patients with 30 disorders: It was diagnostic in 9 disorders, narrowed down the diagnostic possibilities in 14 disorders, and revealed important but non-diagnostic abnormalities, e.g. cerebellar atrophy in 7 disorders. Having a normal magnetic resonance imaging scan was mostly seen in genetic diseases or in the early course of ataxia telangiectasia. Repeat neuroimaging, performed in 108 patients, was generally helpful in monitoring disease evolution and in making a diagnosis. Neuroimaging was not directly helpful in 36 patients with 10 disorders or by definition the 55 patients with unknown disease etiology. Conclusions: Normal or abnormal neuroimaging findings and repeat neuroimaging are very valuable in the diagnosis and management of disorders associated with pediatric chronic ataxia.
\end{abstract}

RÉSUMÉ: Résultats en neuro-imagerie et utilité d'examens répétés en neuro-imagerie dans les cas d'ataxie chronique chez l'enfant. Contexte: L'ataxie chronique, dont la durée excède deux mois, est un phénomène relativement fréquent dans la pratique clinique en neurologie pédiatrique et représente un défi en matière de diagnostic. Elle est causée par plusieurs différents types de désordres. Notre objectif est ici de décrire les divers aspects de la neuro-imagerie et l'utilité d'examens répétés en neuro-imagerie dans les cas d'ataxie chronique chez l'enfant, et ce, afin de déterminer leur apport en ce qui concerne le diagnostic et le suivi de cette pathologie. Matériel et méthodes: Nous avons passé en revue des récapitulatifs graphiques ainsi que des rapports en neuro-imagerie concernant 177 enfants atteints d'ataxie chronique. Les examens en neuro-imagerie de 130 patients sur 177 ont également été analysés. Résultats: Dix-neuf patients n'ont passé qu'un examen de tomographie assistée par ordinateur; 103 d'entre eux n'ont passé qu'un examen d'imagerie par résonnance magnétique (IRM); enfin, 55 ont été soumis à ces deux examens. Des anomalies dans le cervelet et d'autres régions du cerveau ont été associées à l'ataxie. La neuro-imagerie s'est révélée utile dans le cas de 73 patients atteints de 30 désordres: ainsi, elle a permis le diagnostic formel de 9 désordres; elle a permis d'exclure des possibilités de diagnostic dans le cas de 14 désordres; elle a enfin permis de révéler d'importantes anomalies hors diagnostic, par exemple l'atrophie cérébelleuse dans le cas de 7 désordres. Le fait de passer des examens habituels d'IRM a été noté la plupart du temps dans le cas de maladies génétiques ou en ce qui regarde des cas d'ataxie télangiectasie encore à un stade précoce. Répéter des examens en neuro-imagerie chez 108 patients s'est avéré dans l'ensemble utile pour surveiller l'évolution des désordres et établir un diagnostic. La neuro-imagerie n'est pas apparue directement utile dans le cas de 36 patients atteints de 10 désordres ou, par définition, de 55 patients dont l'étiologie demeurait inconnue. Conclusions: Que les résultats en neuro-imagerie soient normaux ou anormaux, ils demeurent très utiles, à l'instar des examens qu'on répète, dans le diagnostic et le suivi des désordres associés à l'ataxie chronique chez l'enfant.

Keywords: Chronic ataxia, pediatric, MRI

doi:10.1017/cjn.2016.7

Can J Neurol Sci. 2016; 43: 824-832

Children with coordination difficulties are encountered relatively commonly in pediatrics. Incoordination of the limbs, poor balance, and unstable stance not caused by decreased level of consciousness, spasticity, muscle weakness, extrapyramidal movement disorder, skeletal disease, or psychogenic disorder constitute ataxia. ${ }^{1}$ Ataxia is caused by many disorders affecting the central or peripheral nervous system or both. ${ }^{2,3}$

When a brain magnetic resonance image (MRI) is not available on urgent basis, a head computed tomography (CT) scan is useful in the assessment of acute and subacute ataxia, especially for

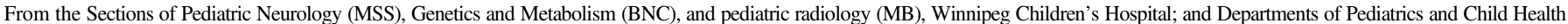
(MSS, BNC), Biochemistry and Medical Genetics (BNC), and Radiology (MB), College of Medicine, Faculty of Health Sciences, University of Manitoba, Winnipeg, Manitoba, Canada. Received March 9, 2015. Final Revisions Submitted December 17, 2015.

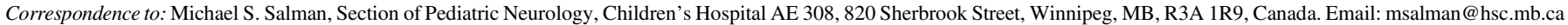


excluding brain hemorrhage, hydrocephalus or brain tumours. However, a brain MRI is considered to be the most appropriate imaging modality in such patients. ${ }^{3-5}$

Several neuroimaging patterns have been described in disorders that cause ataxia. ${ }^{1,4-6}$ Abnormal neuroimaging findings may be isolated to the cerebellum, involve supra- and infratentorial structures e.g. infantile neuronal ceroid lipofuscinosis or may affect the spinal cord e.g. Friedreich ataxia. ${ }^{3}$ Infratentorial abnormalities usually affect the vermis with or without the cerebellar hemispheres. Brainstem abnormalities may be seen, especially in the pons e.g. congenital disorder of glycosylation type $1 a^{6}{ }^{6}$ Several types of neuroimaging abnormalities can be detected depending on the disease process including atrophy, hypoplasia, both atrophy and hypoplasia, infarct, hemorrhage, and dysplasia (disorganized development of infratentorial and sometimes supratentorial structures). ${ }^{6}$ In some disorders, abnormal signal intensity within the cerebellum may add further clues to the diagnosis e.g. infantile neuroaxonal dystrophy. ${ }^{6}$ Contrast enhancement may be noted in infective and inflammatory disorders e.g. multiple sclerosis. ${ }^{1}$ Calcification, best detected on head CT, may also aid in the diagnosis e.g. Cockayne syndrome. ${ }^{4}$

The neuroimaging findings in pediatric ataxia have mostly been described in reviews, ${ }^{1,3,4}$ single case reports, or small case series rather than on a large cohort of pediatric patients. One study focused on posterior fossa imaging in children with ataxia. ${ }^{6}$ No studies have systematically and comprehensively described neuroimaging findings in a large cohort of pediatric patients with chronic ataxia in Canada. Furthermore, there is minimal information on the value of repeat neuroimaging. The aims of this study were to systematically describe the spectrum of neuroimaging findings in a large cohort of pediatric patients with chronic ataxia to investigate their contribution in the assessment of these patients and to ascertain if any specific features (or lack of) can aid the assessment, diagnosis, or prioritize the list of diagnostic possibilities. In addition, the value of repeat neuroimaging was investigated. We hypothesized that normal or abnormal neuroimaging findings are valuable in the assessment of pediatric chronic ataxia and that repeat imaging may contribute important information for the diagnosis and management in some patients.

\section{METHODS}

Previously, we identified patients who developed ataxia when they were less than 17 years old between 1991-2008, from several hospital resources and databases. We then selected patients with chronic ataxia only, which we defined as ataxia lasting longer than two months or if it was recurrent. ${ }^{2}$ All patients attended Winnipeg Children's Hospital for an assessment of their ataxia. Patients from neighboring provinces who attended Winnipeg Children's Hospital were also included. Further information on the ascertainment of the patients is described in detail elsewhere. ${ }^{2} \mathrm{We}$ excluded patients whose ataxia was not clinically prominent and did not affect their daily life. Patients with ataxia caused by primary brain tumours, disorders of the peripheral nerves and vestibular system only were excluded, as were patients with developmental coordination disorder or clumsiness. Details on the epidemiology, ethnicity, and geographic distribution of the cohort have been published. ${ }^{2,7}$

Ethical approval for the study was given by the Research Ethics Board of the University of Manitoba. Demographic information, diagnostic data, and neuroimaging reports were retrieved from the patients' hospital medical charts as described previously. $^{2}$ In addition, neuroimaging studies including head CT and, where available, brain and spinal MRI, were also retrieved and systematically reviewed by a pediatric radiologist with expertise in pediatric neuroimaging (MB). Clinical details were concealed from the radiologist. Brain MRI was acquired on 1.5- or 3-Tesla MRI scanner (GE) using standardized protocol with sagittal T1-weighted, axial/coronal T2-weighted, and coronal fluid-attenuated inversion recovery (FLAIR) images. Supplemental imaging sequences were performed as needed including T2*, DWI, ADC maps, fast spoiled gradient echo (FSPGR) images, and MRA (magnetic resonance angiography). Contrast with gadolinium was given at the discretion of the radiologist. The presence of cerebellar hypoplasia (small size but normal shape), atrophy (shrunken size with prominence of the cerebellar folia), malformation (e.g. Dandy-Walker malformation, molar tooth sign), other structural abnormalities, and signal abnormalities in the cerebellar vermis, cerebellar hemispheres, brainstem, and supratentorial structures including the cortex and white matter, basal ganglia and thalami were recorded.

The frequencies and types of the first and repeat imaging modalities were described for the different disease etiologies. The diagnostic utility of neuroimaging was grouped as follows on retrospective review: A) Extremely helpful because it was diagnostic, B) very helpful in narrowing down the list of diagnostic possibilities but not for making a definitive diagnosis, C) was of limited diagnostic usefulness by revealing important but non-diagnostic abnormalities, and D) not helpful in the diagnosis. Neuroradiological changes on repeat neuroimaging were classified into five categories: Stable (no change), better, worse, fluctuating, or unknown.

Age at first neuroimaging and time period between the first two neuroimaging studies were calculated for each disease etiology. Statistical analyses were conducted using a Statistical Package for the Social Sciences (SPSS) version 22 (IBM, Armonk, NY, USA). Mean and standard deviation were used to describe normally distributed data while median and range were used if the data were skewed.

\section{RESULTS}

\section{Basic demographics}

The cohort of patients with chronic ataxia who satisfied the inclusion criteria consisted of 184 patients (males $=92$ ). The median age (range) at any symptom onset was $1.3(0-16)$ years. The median age (range) at the end of the study was 15 (1-34) years. Detailed demographic information has already been published on this cohort. ${ }^{2}$ Selected presenting symptoms in some of the more common diseases are displayed in Table 1. Four hundred and forty one neuroimaging studies were performed on 177 patients (MRI = 343, CT $=98$ ). Nineteen patients had head CT only, 103 brain MRI only, and 55 had both. Neuroimaging studies in 130 of the 177 patients were reviewed by the radiologist (MB). In the other 47 , neuroimaging findings were extracted from the neuroimaging reports because their imaging studies were not available. The type of neuroimaging and age at first imaging are reported in Supplementary Table 1, which shows that the median age at first imaging varied within and across the different etiologies. Neuroimaging was performed at an earlier age in patients with Angelman syndrome, mitochondrial disorders, and neuronal ceroid lipofuscinosis types 1-3 at one to three years of age; a few years later in ataxia telangiectasia, Rett syndrome, and leukodystrophy; and towards the end of the first decade in Friedreich ataxia. 
Table 1: Selected presenting symptoms in some of the more common diseases

\begin{tabular}{l|c|c|c|c}
\hline Disease & Number of patients & Global developmental delay & Language/speech delay & Seizures \\
\hline Angelman syndrome & 16 & 16 & 0 & 4 \\
\hline Ataxia telangiectasia & 13 & 1 & 2 & 0 \\
\hline Stroke: & & & & \\
\hline 1) All ischemic strokes & 9 & 1 & 0 & 2 \\
\hline 2) Hemorrhagic & 3 & 1 & 1 & 0 \\
\hline Mitochondrial disorders & 9 & 0 & 1 & 2 \\
\hline Friedreich ataxia & 7 & 0 & 0 & 0 \\
\hline Episodic ataxia & 7 & 2 & 1 & 1 \\
\hline Neuronal ceroid lipofuscinosis & 6 & 2 & 0 & 5 \\
\hline Intractable epilepsy syndrome & 5 & 1 & 0 & 5 \\
\hline Hypoxic ischemic encephalopathy following birth & 5 & 4 & & 3 \\
\hline Neuronal migration disorder & 5 & 3 & & 3 \\
\hline Joubert syndrome and related disorders & 5 & 3 & 0 & 0 \\
\hline Rett syndrome & 4 & 2 & 0 & 2 \\
\hline Salla disease & 4 & & & 1 \\
\hline
\end{tabular}

The disorders encountered were divided into eight categories (Supplementary Table 2) : Metabolic diseases (generally caused by enzyme deficiency or transporter defect) (nine disorders), genetic diseases (fifteen disorders), diseases caused by impaired DNA repair (two disorders), intractable epilepsy (one disorder), stroke (six disorders from different causes), inflammatory diseases (three disorders), other acquired diseases (four disorders), and unknown (five subcategories based on the clinical manifestations).

\section{General neuroimaging findings}

Neuroimaging abnormalities in the cerebellum or other brain regions such as the cerebral hemispheres were described in our cohort with chronic ataxia. Neuroimaging findings varied both within and among the various disease etiologies. Brain MRIs of adequate quality for the evaluation of myelin was available in 120 of the 130 patients, whose neuroimaging was reviewed. Myelination was normal in at least 96 patients. Delayed myelination, hypomyelination, or abnormal myelin/ demyelination was seen in the rest and also in some of the patients with normal myelination, due to various disease processes including inflammation, ischemia, neurodegeneration, radiotherapy, and neoplasia. Supplementary Table 2 summarizes the neuroimaging findings of the disorders encountered in our cohort.

Details on the diagnostic utility of neuroimaging is shown in Supplementary Table 3 . Neuroimaging was helpful to a varying extent in 73 patients belonging to the first seven disease categories described above (totaling 30 disorders): A) Imaging was extremely helpful because it was diagnostic in nine diseases (four genetic disorders, five different disorders causing acute stroke with sequelae), B) imaging was very helpful in narrowing down the list of diagnostic possibilities but not for making a definitive diagnosis in 14 diseases (four metabolic disorders, three genetic diseases, one type of stroke, two inflammatory diseases, four acquired disorders), C) imaging was of limited diagnostic usefulness by revealing important but non-diagnostic abnormalities in seven diseases (two disorders caused by impaired DNA repair, four genetic diseases, one inflammatory disease).

There were some patients who did not have diagnostic findings on their neuroimaging despite having disorders with previously well-described neuroimaging abnormalities. Indeed, in five of the 30 disorders where neuroimaging was deemed helpful in our study, there were 13 patients whose neuroimaging was either normal or showed abnormalities not contributing to the diagnosis (Supplementary Table 3).

Neuroimaging was not directly helpful in 36 patients with 10 diseases (five metabolic diseases, four genetic diseases, and one in the intractable epilepsy category) (Supplementary Table 3).

In addition, neuroimaging was not helpful (by definition) in 55 patients whose diagnosis was unknown at the end of our study period, despite extensive investigations available at the time of their assessment. Forty of the 55 patients had a non-progressive disease course. The other 15 patients had intermittent ataxia $(\mathrm{N}=6)$, progressive ataxia with or without developmental regression $(\mathrm{N}=7)$, or self-limiting ataxia that lasted for several months $(\mathrm{N}=2)$. The 40 patients with the non-progressive course were divided into six mutually exclusive subgroups depending on the presence or absence of developmental delay, pyramidal tract signs, and epilepsy as described previously. ${ }^{7}$ Their neuroimaging features were variable overall and also within their respective clinical subgroups. Some had normal imaging, while others had cerebellar hypoplasia, or progressive cerebellar atrophy despite the absence of clinical progression. Similarly, variable neuroimaging features were noted in the 15 patients of 55 patients with unknown diagnosis (Supplementary Table 2).

Neuroimaging review resulted in etiological re-classification of seven patients from our original cohort following the discovery of neuroimaging findings that were not fully appreciated previously, ${ }^{2}$ or following the re-evaluation of previously described 
findings. These seven patients had stroke, neuronal migration abnormalities, leukodystrophy, and Joubert syndrome related disorders (JSRD).

\section{Repeat neuroimaging}

Repeat neuroimaging ( $\mathrm{MRI}=288, \mathrm{CT}=84$ ) was performed once or more in 108 of $177(61 \%)$ patients based on clinical indication (Supplementary Table 4). The median time between the first two scans was 1.2 years (range: same day-21.4 years). The types of the first two scans were as follows: Fifty three patients had MRI scan first followed by another MRI after a median of 1.9 years (range: 2 days-8.2 years), 39 had CT first followed by MRI after a median of 0.8 years (range: same day-21.4 years), 11 had CT first followed by another CT after a median of 1.5 years (range: 1 day-9 years), and five had MRI first followed by CT after a median of 0.7 years (range: 14 days-7.3 years).

Among disease etiologies with at least five patients who had repeat neuroimaging, all (or almost all) patients with stroke, mitochondrial disorder, neuronal ceroid lipofuscinosis, intractable epilepsy, and hypoxic ischemic encephalopathy at birth had repeat neuroimaging, mostly MRI; while a smaller proportion of patients with Angelman syndrome (6 of 15 who had imaging) and ataxia telangiectasia (6 of 11 who had imaging) had repeat neuroimaging.

The time period between the first two scans varied among the different etiologies (Supplementary Table 5). Patients with stroke and neuronal ceroid lipofuscinosis had repeat neuroimaging sooner (median times of 0.4 and 0.85 years respectively); while patients with mitochondrial disorder, Angelman syndrome, ataxia telangiectasia, and intractable epilepsy had their neuroimaging later (median times ranging between 1.05 and 2.32 years); and finally patients with and hypoxic ischemic encephalopathy at birth had their repeat neuroimaging after a median period of 4.64 years (Supplementary Table 5).

Repeat neuroimaging showed no changes in 41 patients, improvement in nine, worsening in 23 , fluctuating changes in eight, and was unknown in 27 (Supplementary Table 4). In patients with progressive disorders e.g. ataxia telangiectasia, repeat neuroimaging showed no changes or worsening; while in neuronal ceroid lipofuscinosis, leukodystrophy, and Friedreich ataxia worsening on repeat neuroimaging was uniformly seen. In patients with stroke and mitochondrial disorders, changes on neuroimaging were more variable with some showing improvement or no changes, while in others worsening was noted. In patients with non-progressive clinical disorders such as JSRD, hypoxic ischemic encephalopathy at birth, neuronal migration disorder, or in patients with Rett syndrome, and several other rare genetic and metabolic disorders, imaging features were generally stable on repeat imaging.

\section{Neuroimaging findings in specific diseases}

There were 16 patients with Angelman syndrome. Delayed myelination was described in three. Thirteen patients had ataxia telangiectasia. Three presented with brain tumours and three had cerebellar atrophy. Nine patients had ischemic stroke associated with chronic ataxia from multiple causes (Supplementary Table 2). Typical imaging features consistent with infarcts were found in brain regions supplied by the posterior cerebral circulation (Figure 1). In addition, two other patients had ischemic thrombo-embolic infarcts due to pneumococcal septicemia and cardiomyopathy in association with Friedreich ataxia. One patient with Gaucher disease type 3 had a small hemorrhage (not related to the patient's ataxia) caused by a small cavernoma in the left caudate head.

The nine patients with mitochondrial disorders had abnormalities in respiratory chain enzyme complexes I (one), IV (one), or both I and IV (two), pyruvate carboxylase deficiency (one), MELAS (mitochondrial encephalomyopathy, lactic acidosis and stroke-like episodes) (one), Leigh syndrome (one), Alpers disease (one) (Figure 2), and an uncharacterized mitochondrial disorder (one). MRI was normal in one and abnormal in the rest with hyperintense signal abnormalities in several brain regions including cerebral white matter and cortical and deep grey matter on T2-weighted images (Supplementary Table 2).

Seven patients had Friedreich ataxia, three of whom had spinal cord atrophy (Figure 3). Six patients had neuronal ceroid lipofuscinosis types 1-3 (Supplementary Figure 1, Supplementary Table 2). Diffuse brain atrophy and progressive imaging changes were seen in five patients. There were five patients with neuronal migration disorders causing diffuse or focal e.g. parietal-occipital lobes pachygyria or polymicrogyria (Supplementary figure 2). Neuroimaging was not diagnostically helpful in the five patients with intractable epilepsy. Abnormalities in signal intensity, restricted diffusion, and atrophy were seen in the cerebral white and grey matter, suggestive of cytotoxic edema with or without vasogenic edema.

Four patients had Salla disease and all showed hypomyelination (Supplementary figure 3). Five patients had a JSRD. All had the molar tooth sign on MRI (Supplementary figure 4).

Table 2 shows important MRI features that may give the clinician clues in the diagnosis of patients with chronic ataxia. The list is not comprehensive and is based on the various diseases encountered in our cohort. Of interest is that hypointense signal abnormalities in the thalami and globi pallidi were seen in patients with neuronal ceroid lipofuscinosis, while hypointense signal abnormality in the thalami associated with hyperintense signal abnormality in the basal ganglia were seen in the single patient with post-radiation ataxia.

\section{Discussion}

Following a detailed clinical assessment of patients with ataxia, the next step is usually neuroimaging. Neuroimaging is essential in excluding diseases amenable to treatment that may present with ataxia such as posterior fossa tumour, hemorrhage, abscess, or hydrocephalus.

The neuroimaging abnormalities seen in our cohort revealed that pediatric chronic ataxia is associated not only with abnormalities in the cerebellum but also with abnormalities in several other brain regions such as the cerebral hemispheres. This could reflect the widespread connectivity of cerebellar neural networks. Neuroimaging review resulted in few etiological re-classification of the original diagnoses; specifically strokes, neuronal migration disorders, leukodystrophy, and cerebellar malformations. This is not surprising since significant advances in neuroimaging techniques and medical knowledge has taken place since 1991, the start date of the current investigation.

\section{Normal or non-specific abnormalities on initial neuroimaging}

Having a normal MRI can be helpful since it excludes disorders belonging to several disease categories that are associated with ataxia e.g. brain malformations, demyelination, or 

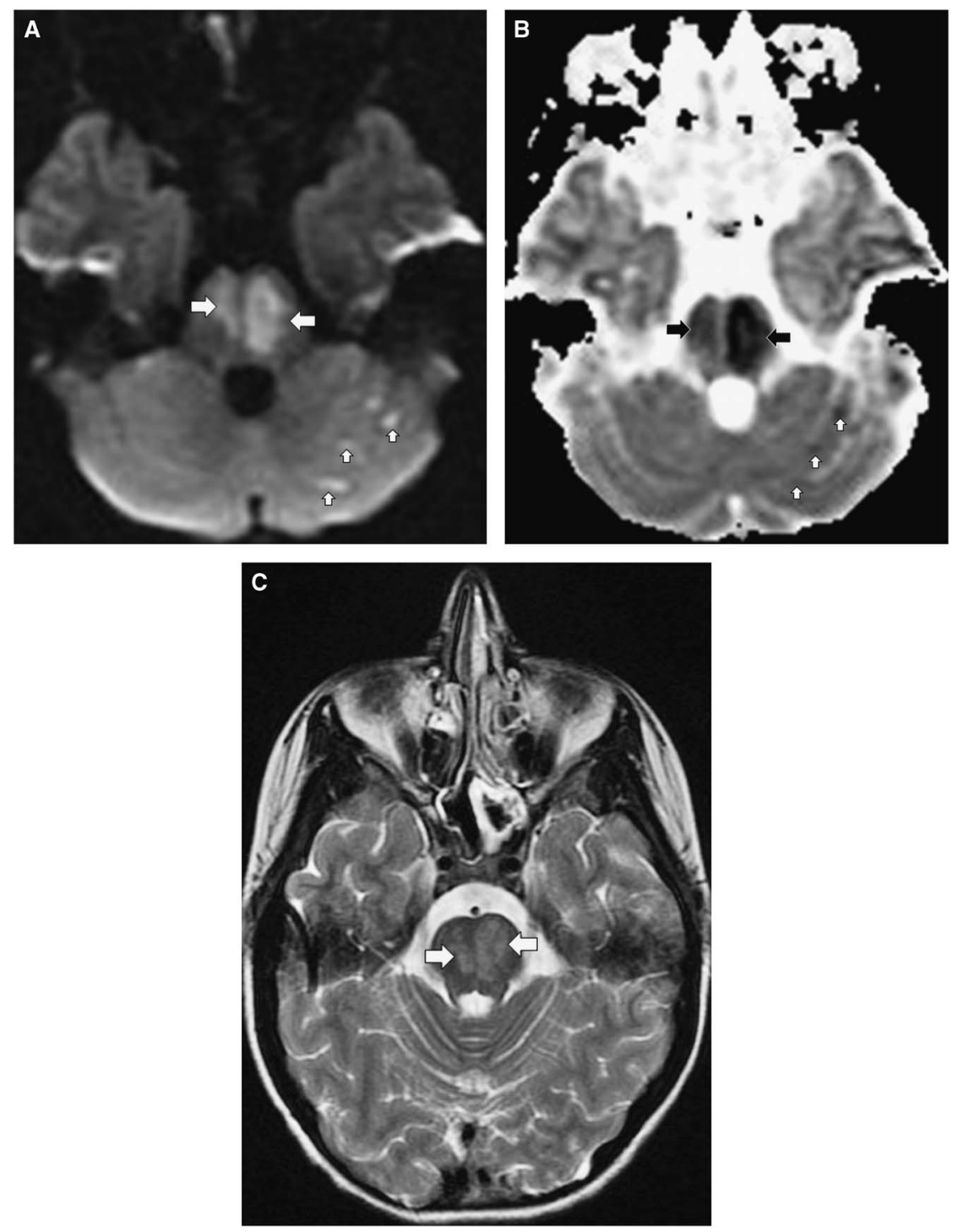

Figure 1: Brain MRI of a 16-year old female with pontine and left cerebellar hemisphere infarction. A) Axial DWI (TR 8000, TE 79, slice thickness $5 \mathrm{~mm}$ ) and B) Axial ADC map (TR 8000, TE 79, slice thickness $5 \mathrm{~mm}$ ) show hyperintense signal abnormalities in the pons, left more than right (horizontal arrows) and in few patchy areas in the left cerebellar hemisphere (vertical arrows) in (A) with matching abnormalities in $(B)$. The findings represent restricted diffusion due to cytotoxic edema and are consistent with ischemic arterial infarction in the distribution of the perforating pontine branches of the basilar artery and left posterior inferior cerebellar artery territories. C) Axial T2WI (TR 2317, TE 13, slice thickness $6 \mathrm{~mm}$ ) shows hyperintense signal abnormality in the pons (arrows).

hemorrhages. In addition, a normal conventional MRI (or one with non-specific findings) is useful, especially when it remains normal on repeat imaging, since it is mostly associated with certain genetic diseases that cause chronic ataxia e.g. Rett syndrome, Angelman syndrome, Glut1 deficiency, and episodic ataxia type 2 . This is consistent with findings reported in other studies. ${ }^{1,8-10}$ In episodic ataxia type 2 cerebellar atrophy may develop in some patients, usually beyond the pediatric age group.

It is worth noting that delayed myelination and increased ventricular size on brain MRI in children with Angelman syndrome, the commonest etiology in our cohort, has been reported previously. ${ }^{9}$ A few of our patients with Angelman syndrome had these so-called 'non-specific findings'. Some patients with Rett syndrome have been reported to show cerebral atrophy or mild cerebellar tissue loss on conventional MRI, which was confirmed on volumetric MRI studies. ${ }^{10}$ This was not seen in our two patients with Rett syndrome with repeat brain MRI.

A normal MRI was seen in the early course of some patients with ataxia telangiectasia. This should be kept in mind when assessing young children presenting with ataxia or dystonia, who in their early disease course may not have developed telangiectasia or abnormalities in saccade initiation 

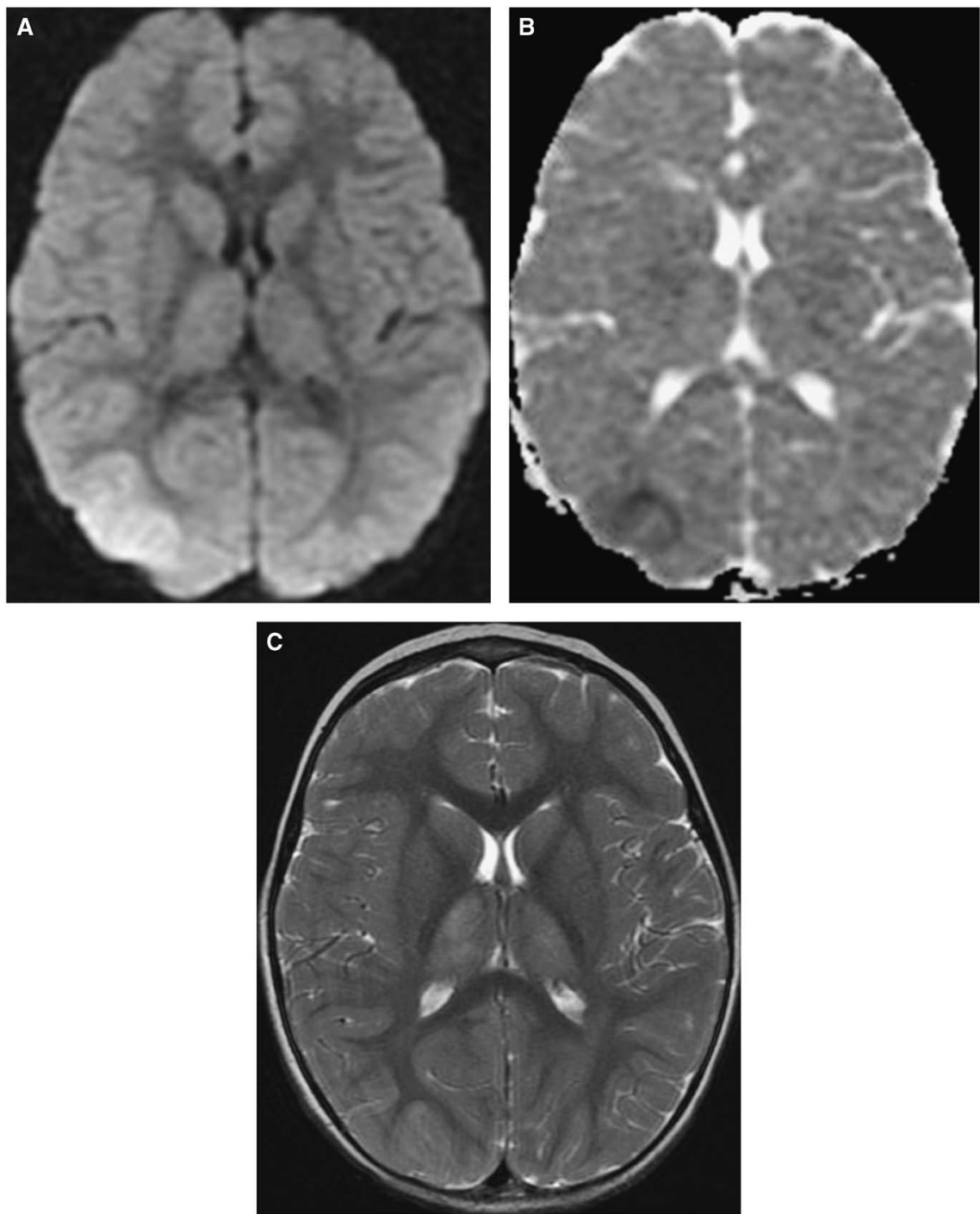

Figure 2: Brain MRI in a 30-months old girl with Alpers disease (progressive neuronal degeneration of childhood). A) Axial DWI (TR 8000, TE 79.9, slice thickness $5 \mathrm{~mm}$ ) and B) Axial ADC map (TR 8000, TE 79.9, slice thickness $5 \mathrm{~mm}$ ) show hyperintense signal abnormality in the right parieto-occipital lobe region in (A) with matching abnormality in $(B)$. The findings represent restricted diffusion and are consistent with stroke-like lesion. C) Axial T2WI (TR 2350, TE 111.4, slice thickness $6 \mathrm{~mm}$ ) shows hyperintense signal abnormality in the thalami. Atrophy of the thalami was seen on subsequent scans (not shown).

that usually give away their ataxia telangiectasia diagnosis. Cerebellar atrophy is documented to appear later in the course of the disease, ${ }^{8}$ as we observed on repeat imaging in some of our patients.

In addition, normal or non-specific abnormalities on initial neuroimaging may be seen in diseases that are usually known to have helpful diagnostic neuroimaging features, e.g. in mitochondrial disorders. This may be due to the variable nature of disease manifestation or severity among patients or may occur when neuroimaging is done early in the course of the disease. A repeat MRI is warranted when the index of suspicion is high.

Microscopic lesions are beyond conventional MRI resolution. Hence, extensive Purkinje cell dropout may not result in cerebellar abnormalities on MRI.

\section{Neuroimaging abnormalities in pediatric chronic ataxia}

A Swedish study published in 1996 on 78 children with non-progressive ataxia, ${ }^{11}$ revealed that $61 \%$ of 70 children with adequate neuroimaging had normal scans. However, twenty-nine patients had CT scan only. The authors stated that MRI added new information only when the CT showed an abnormality. However, we are aware of many instances, where a CT scan missed abnormalities that were only seen on an MRI performed within hours of the CT, such as demyelinating lesions or cerebellar vermis hypoplasia. The abnormalities reported in the Swedish study and few other studies, ${ }^{6,12-15}$ revealed similar neuroimaging abnormalities seen in our patients with non-progressive ataxia including neuronal migration disorders, JSRD, Dandy-Walker 

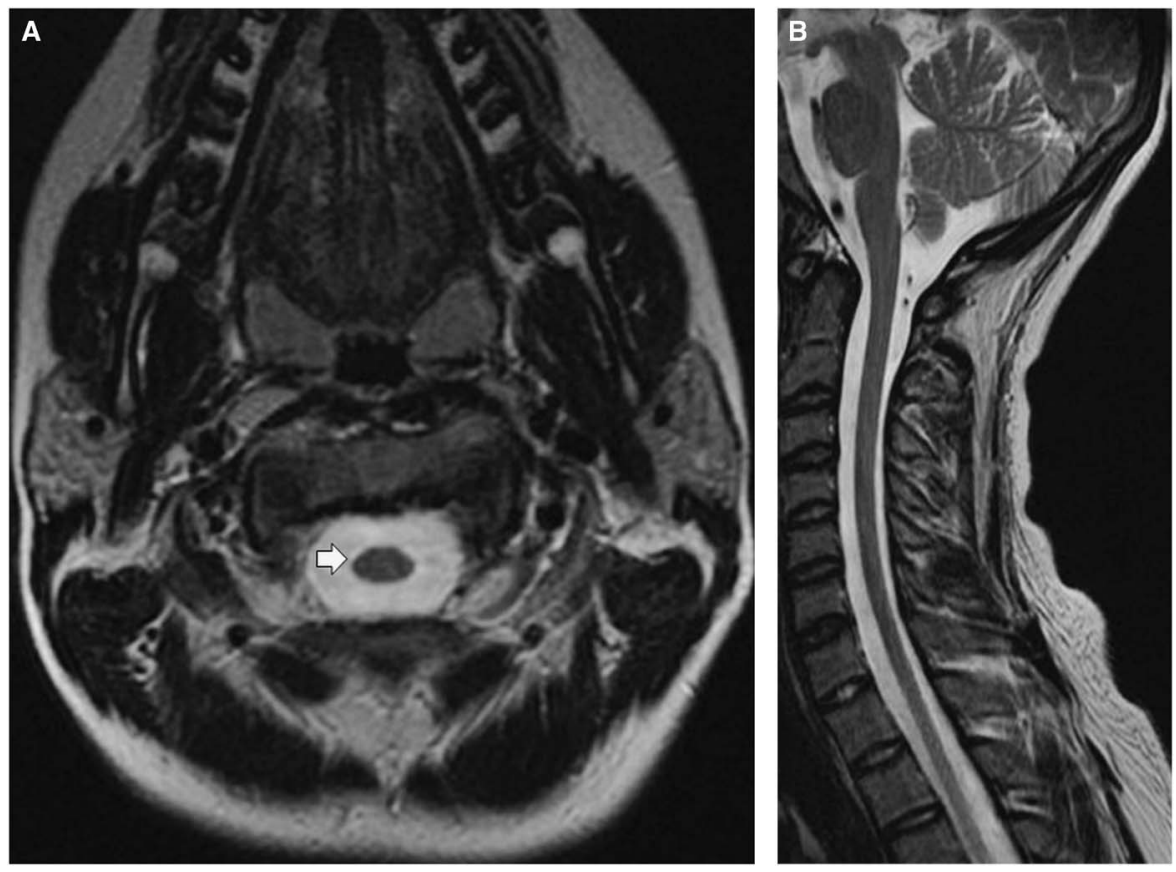

Figure 3: Spinal cord MRI of a 16-year old female with Friedreich ataxia. A) Axial T2WI of the cervical spinal cord (TR 5967, TE 114.2, slice thickness $5.5 \mathrm{~mm}$ ) and B) Midsagittal T2WI of the cervical and upper thoracic spinal cord (TR 3617, TE 112, slice thickness $4 \mathrm{~mm}$ ) show thinning of the spinal cord consistent with atrophy, which is more easily appreciated on the axial image (arrow) in $(A)$.

malformation, cerebellar hypoplasia, and nonspecific findings such as arachnoid cysts and mega cisterna magna.

The neuroimaging pattern in patients with ataxia telangiectasia, ${ }^{4,8,14,16}$ Friedreich ataxia, ${ }^{5,8,16}$ and the various subtypes of mitochondrial diseases, ${ }^{5,6,8,14,17}$ stroke, ${ }^{17}$ especially those involving posterior fossa structures, ${ }^{1,4,18,19}$ neuronal ceroid lipofuscinosis, ${ }^{8,14}$ hypoxic ischemic encephalopathy, ${ }^{17}$ Salla disease, ${ }^{8,20}$ adrenoleukodystrophy, ${ }^{5,8}$ prematurity, ${ }^{17,21}$ acute

\section{Table 2: Diagnostic MRI clues in diseases associated with pediatric chronic ataxia}

\begin{tabular}{|c|c|}
\hline Neuroimaging features & Disease \\
\hline \multicolumn{2}{|l|}{ 1) Supratentorial abnormalities ( \pm infratentorial abnormalities): } \\
\hline \multicolumn{2}{|l|}{ A) Abnormal signal intensity in grey or white matter or both on T2-weighted or FLAIR MRI: } \\
\hline i) Symmetrical or asymmetrical & $\begin{array}{l}\text { Mitochondrial, neuronal ceroid lipofuscinosis, leukodystrophy, hypoxic-ischemic } \\
\text { encephalopathy following birth, prematurity, intractable epilepsy, stroke }\end{array}$ \\
\hline ii) Multifocal & acute disseminated encephalomyelitis, multiple sclerosis, post radiation, vasculitis \\
\hline B) Structural abnormalities & $\begin{array}{l}\text { neuronal migration disorder, dysequilibrium syndrome (also known as autosomal recessive } \\
\text { cerebellar hypoplasia in the Hutterite population), hypoxic-ischemic encephalopathy } \\
\text { following birth, prematurity, trauma }\end{array}$ \\
\hline C) Hypomyelination & Salla disease, other leukodystrophies, Mitochondrial disorders (rarely) \\
\hline D) Delayed myelination & Angelman syndrome (in some patients) \\
\hline $\begin{array}{l}\text { 2) Unilateral or multiple infra- and/or supratentorial abnormalities consistent with } \\
\text { infarcts or hemorrhages involving posterior circulation territories, } \pm \text { abnormal MRA }\end{array}$ & Stroke (thrombo-embolic ischemic, aneurysm, AVM, cavernoma) \\
\hline \multicolumn{2}{|l|}{ 3) Cerebellar malformations: } \\
\hline A) With brainstem malformation & Joubert syndrome related disorders (molar tooth sign), pontocerebellar hypoplasia type 3 \\
\hline B) Without brainstem malformation & Dandy-Walker malformation \\
\hline 4) Spinal cord atrophy & Friedreich ataxia \\
\hline 5) Normal & $\begin{array}{l}\text { Episodic ataxia, GLUT1 deficiency, Rett syndrome, Angelman syndrome, ataxia } \\
\text { telangiectasia (initially). }\end{array}$ \\
\hline
\end{tabular}

FLAIR = fluid-attenuated inversion recovery $\mathrm{MRI}=$ Magnetic resonance imaging, $\mathrm{MRA}=$ magnetic resonance angiography, $\mathrm{AVM}=$ arteriovenous malformation 
disseminated encephalomyelitis (ADEM), ${ }^{1}$ multiple sclerosis, ${ }^{1}$ and uncommon syndromes such as Marinesco-Sjögren syndrome, ${ }^{5,8}$ autosomal recessive cerebellar hypoplasia in the Hutterites population (dysequilibrium syndrome), ${ }^{22}$ pontocerebellar hypoplasia type $3,{ }^{23}$ post infectious ataxia (associated only rarely with cerebellar atrophy), ${ }^{8}$ post traumatic ataxia, ${ }^{8}$ and post radiation ataxia, ${ }^{8}$ have been described previously. Patients in our cohort with these diagnoses displayed similar findings (Supplementary Table 2). In general, abnormal signal intensity on brain MRI involving the supratentorial regions tends to point to a metabolic or an inflammatory etiology, while a normal brain MRI points to genetic or rare metabolic disorders.

Patients with severe intractable epilepsy had no specific diagnosis despite multiple investigations. All had multiple types of seizures that at times occurred on daily basis. All had developmental delay and/ or learning difficulties. Their clinical course was consistent with an epileptic encephalopathy. Their ataxia was present during periods of good seizure control. None had brain malformations or neuronal migration disorders on MRI. Their brain MRI showed non-diagnostic abnormalities that may or may not be related to their frequent seizures or underlying disease. Reversible and irreversible MRI findings similar to those reported in our patients including signal intensity changes, restricted diffusion, atrophy, or structural abnormalities have been described in patients with severe epilepsy and status epilepticus. ${ }^{24-26}$

The typical MRI features described in patients with Wernicke encephalopathy, i.e. abnormal signal intensity over the medial thalami, mammillary bodies, tegmentum, periaqueductal and tectal plate, ${ }^{4,27}$ are not universally seen in these patients. ${ }^{27}$ Our single patient with Wernicke encephalopathy did not have these abnormalities either.

\section{Repeat neuroimaging}

Indications for repeat neuroimaging were variable and depended on clinical need. Repeat neuroimaging was helpful in monitoring disease evolution and progression over time by revealing changes in previous findings or showing new findings e.g. cerebral and cerebellar atrophy in neuronal ceroid lipofuscinosis, cerebellar atrophy in ataxia telangiectasia, and demyelinating lesions in multiple sclerosis, which were not necessarily associated with clinical progression. In some instances repeat neuroimaging provided further assurance to the physician and family; for example, by revealing improvement in patients with central nervous system vasculitis following immunosuppressive treatment, resolution of brain hemorrhage in cavernoma, or by confirming a stable neuroimaging course in hypoxic ischemic encephalopathy following birth, Chiari 1 malformation, and some genetic syndromes. The timing of repeat neuroimaging was variable. Repeat neuroimaging should be tailored individually.

\section{Study limitations}

Our retrospective study limitations include missing, incomplete and inaccurate information recorded in the hospital charts. Actual neuroimaging studies were not available despite extensive search in 47 of 177 patients. These were mostly from patients who presented in the early 1990's. In addition, 19 patients from the earlier study period only had CT imaging performed, which is not ideal for imaging the brain because of its inferior spatial resolution, especially for posterior fossa structures in comparison to brain MRI. Additional limitations include the variable MRI sequences used over the study period and the long time range of the study during which many medical advances and discoveries were made, as discussed elsewhere. ${ }^{2}$ Although the age range of the cohort at the end of the study period is wide, all patients were in the pediatric age range when they developed ataxia.

\section{Conclusions and future directions}

Central nervous system imaging with MRI is very valuable and important investigation in the assessment of pediatric chronic ataxia. Consideration should be given to repeating MRI in patients with chronic ataxia who remain without a diagnosis despite appropriate clinical evaluation and investigations.

Other more advanced neuroimaging modalities have been widely utilized in patients with ataxia and/ or cerebellar disorders, e.g. functional MRI, diffusion tensor imaging (tractography), positron emission tomography and single-photon emission computed tomography. ${ }^{4,28}$ However, their role has not been fully evaluated and their routine use is not recommended, since their contribution to the management of individual patients with cerebellar disorders remains unclear. ${ }^{3,28}$ They may be helpful in certain circumstances, for example inflammatory or paraneoplastic disorders of the cerebellum. ${ }^{4,28}$ The role of diffusion tensor imaging is currently being evaluated and its future potential appears promising especially in patients with posterior fossa malformations. ${ }^{29}$

Future studies should investigate patients with non-progressive chronic ataxia, who remain without a diagnosis despite extensive investigations, using whole exome sequencing if their microarray test is negative. Furthermore, more research is needed in ataxic patients with non-progressive disease course but who show progressive cerebellar atrophy pattern on MRI.

\section{ACKNowledgements ANd Grant SuPPort}

The authors thank the Health Science Centre Foundation and Branden's Hope Fund for their financial support; The Children's Hospital Research Institute of Manitoba and The Children Hospital Foundation of Manitoba for providing the infrastructure needed for the project and for financial support; Dr. S. Masood for performing part of the data collection and Dr. A. Poretti for his critical review of the manuscript.

\section{Disclosures}

Michael Salman, Bernard Chodirker, and Martin Bunge do not have anything to disclose.

\section{SUPPLEMENTARY MATERIAL}

To view supplementary material for this article, please visit http://dx.doi.org/10.1017/cjn.2016.7

\section{REFERENCES}

1. Poretti A, Benson JE, Huisman TA, Boltshauser E. Acute ataxia in children: approach to clinical presentation and role of additional investigations. Neuropediatrics. 2013;44:127-41.

2. Salman MS, Lee EJ, Tjahjadi A, Chodirker BN. The epidemiology of intermittent and chronic ataxia in children in Manitoba, Canada. Dev Med Child Neurol. 2013;55:341-7. 
3. Brunberg JA. Expert Panel on Neurologic Imaging. Ataxia. AJNR Am J Neuroradiol. 2008;29:1420-2.

4. Mascalchi M, Vella A. Magnetic resonance and nuclear medicine imaging in ataxias. Handb Clin Neurol. 2012;103:85-110.

5. Vedolin L, Gonzalez G, Souza CF, Lourenço C, Barkovich AJ. Inherited cerebellar ataxia in childhood: a pattern-recognition approach using brain MRI. AJNR Am J Neuroradiol. 2013;34:925-34; S1-2.

6. Boddaert N, Desguerre I, Bahi-Buisson N, et al. Posterior fossa imaging in 158 children with ataxia. J Neuroradiol. 2010;37:220-30.

7. Salman MS, Masood S, Azad M, Chodirker BN. Ethnicity and geographic distribution of pediatric chronic ataxia in Manitoba. Can J Neurol Sci. 2014;41:29-36.

8. Poretti A, Wolf NI, Boltshauser E. Differential diagnosis of cerebellar atrophy in childhood. Eur J Paediatr Neurol. 2008;12:155-67.

9. Harting I, Seitz A, Rating D, et al. Abnormal myelination in Angelman syndrome. Eur J Paediatr Neurol. 2009;13:271-6.

10. Dunn HG, MacLeod PM. Rett syndrome: review of biological abnormalities. Can J Neurol Sci. 2001;28:16-29.

11. Esscher E, Flodmark O, Hagberg G, Hagberg B. Non-progressive ataxia: origins, brain pathology and impairments in 78 Swedish children. Dev Med Child Neurol. 1996;38:285-96.

12. Gleeson JG, Keeler LC, Parisi MA, et al. Molar tooth sign of the midbrain-hindbrain junction: occurrence in multiple distinct syndromes. Am J Med Genet A. 2004;125A:125-34.

13. Shekdar K. Posterior fossa malformations. Semin Ultrasound CT MR. 2011;32:228-41.

14. Al-Maawali A, Blaser S, Yoon G. Diagnostic approach to childhoodonset cerebellar atrophy: a 10-year retrospective study of 300 patients. J Child Neurol. 2012;27:1121-32.

15. Kanekar S, Gent M. Malformations of cortical development. Semin Ultrasound CT MR. 2011;32:211-27.

16. Anheim M, Fleury M, Monga B, et al. Epidemiological, clinical, paraclinical and molecular study of a cohort of 102 patients affected with autosomal recessive progressive cerebellar ataxia from Alsace, Eastern France: implications for clinical management. Neurogenetics. 2010;11:1-12.

17. Rasalkar DD, Chu WC. Imaging in children presenting with acute neurological deficit: stroke. Postgrad Med J. 2012;88:649-60.
18. Mackay MT, Prabhu SP, Coleman L. Childhood posterior circulation arterial ischemic stroke. Stroke. 2010;41:2201-9.

19. Yock-Corrales A, Mackay MT, Mosley I, Maixner W, Babl FE. Acute childhood arterial ischemic and hemorrhagic stroke in the emergency department. Ann Emerg Med. 2011;58:156-63.

20. Steenweg ME, Vanderver A, Blaser S, et al. Magnetic resonance imaging pattern recognition in hypomyelinating disorders. Brain. 2010;133:2971-82.

21. Limperopoulos $\mathrm{C}$, du Plessis AJ. Injury to the Developing Cerebellum: Mechanisms and Consequences. Neoreviews. 2007; 8:e409-17.

22. Boycott KM, Flavelle S, Bureau A, et al. Homozygous deletion of the very low density lipoprotein receptor gene causes autosomal recessive cerebellar hypoplasia with cerebral gyral simplification. Am J Hum Genet. 2005;77:477-83.

23. Rajab A, Mochida GH, Hill A, et al. A novel form of pontocerebellar hypoplasia maps to chromosome 7q11-21. Neurology. 2003; 60:1664-7.

24. Cianfoni A, Caulo M, Cerase A, et al. Seizure-induced brain lesions: A wide spectrum of variably reversible MRI abnormalities. Eur J Radiol. 2013;82:1964-72.

25. Cartagena AM, Young GB, Lee DH, Mirsattari SM. Reversible and irreversible cranial MRI findings associated with status epilepticus. Epilepsy Behav. 2014;33:24-30.

26. Johnston JM, Smyth MD, McKinstry RC. Basics of neuroimaging in pediatric epilepsy. In: Pellock JM, Bourgeois BFD, Dodson WE, editors. Pediatric epilepsy: Diagnosis and therapy. 3rd ed. New York: Demos Medical Publishing; 2008. pg. 213-26.

27. Cerase A, Rubenni E, Rufa A, et al. CT and MRI of Wernicke's encephalopathy. Radiol Med. 2011;116:319-33.

28. Baldarçara L, Currie S, Hadjivassiliou M, et al. Consensus paper: radiological biomarkers of cerebellar diseases. Cerebellum. 2015;14:175-96.

29. Chokshi FH, Poretti A, Meoded A, Huisman TA. Normal and abnormal development of the cerebellum and brainstem as depicted by diffusion tensor imaging. Semin Ultrasound CT MR. 2011;32:539-54. 